\title{
Numerical Simulation of Flow around a Train Passing Through a Tornado
}

\author{
Kohei Obara ${ }^{1}$, Sinisa Krajnovic ${ }^{2}$, Guglielmo Minelli ${ }^{2}$, Nobuyuki Okura ${ }^{1}$, Masahiro Suzuki ${ }^{1}$ \\ ${ }^{1}$ Department of Vehicle and Mechanical Engineering, Meijo University \\ Nagoya, Japan \\ 153436007@ccalumni.meijo-u.ac.jp \\ ${ }^{2}$ Division of Fluid Dynamics, Department of Applied Mechanics, Chalmers University of Technology \\ Gothenburg, Sweden
}

\section{Extended Abstract}

A tornado is one of the most powerful weather phenomena. It has high wind velocity and often brings serious damages to our lives. It also might has power to overturn trains. Actually at least three train overturn accidents in Japan were probably caused by tornadoes. Although numerous studies have been conducted to clarify the nature of tornado and its effects on civil engineering structures, only a few studies have been examined the effects of the tornado on moving vehicles [1]. Thus we investigated the flow around a train passing through a tornado by a numerical simulation.

The compressible Reynolds Averaged Navier-Stokes equations are solved with the k- $\zeta$ - $f$ turbulence model [2] using a commercial finite volume solver, AVL FIRE. A boundary condition was defined based on a set-up of our previous experiment [1]. The maximum tangential velocity and the core radius of the tornado were set at $8 \mathrm{~m} / \mathrm{s}$ and $100 \mathrm{~mm}$, respectively. The swirl ratio was set at 0.8 . A $1 / 40$ scale vehicle was set to travel through the tornado at a speed of $4 \mathrm{~m} / \mathrm{s}$. The Reynolds number based on the maximum tangential velocity and the train width is $3.8 \times 10^{4}$. A deforming and sliding mesh method [3] was employed to achieve a scenario that the train runs through the tornado.

First, in order to check the numerical accuracy, the velocity and the pressure profiles of the tornado itself were compared with those of the experimental data and the analytical model of the tornado - the Rankin vortex model. The results showed good agreements. And then, time histories of the side and lift forces acting on the train were compared with the experimental data. The computational results agreed well with the experimental results. The side force acting on the train changed its direction from negative to positive while passing through the tornado. Finally, the flow field around the train in the tornado were analysed. The analysis showed that the flow field changed moment by moment depending on the position of the train in the tornado and the resulted air forces acting on the vehicle varied.

\section{References}

[1] M. Suzuki, K. Obara and N. Okura, "Development of an experimental method to estimate aerodynamic forces acting on a train in a tornado," in Proceedings of 11th World Congress on Railway Research, Milan, Italy, 2016.

[2] B. Basara, "Eddy viscosity transport model based on elliptic relaxation approach," AIAA Journal, vol. 44, no. 7, pp. 1686-1690, 2006.

[3] D. Uystepruyst and S. Krajnovic, "Numerical simulation of the transient aerodynamic phenomena induced by passing manoeuvres," Journal of Wind Engineering and Industrial Aerodynamics, vol. 114, pp.62-71, 2013. 